Published in final edited form as:

Phys Biol. 2014 April ; 11(2): 026005. doi:10.1088/1478-3975/11/2/026005.

\title{
Comparison of the theoretical and real-world evolutionary potential of a genetic circuit
}

\author{
M. Razo-Mejia ${ }^{1,2}$, J.Q. Boedicker², D. Jones ${ }^{2}$, A. DeLuna ${ }^{3}$, J.B. Kinney ${ }^{4}$, and R. Phillips ${ }^{2}$ \\ R. Phillips: phillips@pboc.caltech.edu \\ ${ }^{1}$ Ingenieria Biotecnologica, Instituto Politecnico Nacional, Av. Mineral de Valenciana No. 200 Col. \\ Fracc. Industrial Puerto Interior, Silao de la Victoria, Guanajuato, 36275, Mexico \\ 2Department of Applied Physics, California Institute of Technology, 1200 East California \\ Boulevard, Pasadena, California 91125, USA \\ ${ }^{3}$ Laboratorio Nacional de Genomica para la Biodiversidad Centro de Investigacion y de Estudios \\ Avanzados del IPN, Km 9.6 Libramiento Norte Carretera Irapuato-Leon, Irapuato, Guanajuato \\ 36821, Mexico \\ ${ }^{4}$ Simons Center for Quantitative Biology, Cold Spring Harbor Laboratory, Cold Spring Harbor, NY \\ 11724
}

\section{Abstract}

With the development of next-generation sequencing technologies, many large scale experimental efforts aim to map genotypic variability among individuals. This natural variability in populations fuels many fundamental biological processes, ranging from evolutionary adaptation and speciation to the spread of genetic diseases and drug resistance. An interesting and important component of this variability is present within the regulatory regions of genes. As these regions evolve, accumulated mutations lead to modulation of gene expression, which may have consequences for the phenotype. A simple model system where the link between genetic variability, gene regulation and function can be studied in detail is missing. In this article we develop a model to explore how the sequence of the wild-type lac promoter dictates the fold change in gene expression. The model combines single-base pair resolution maps of transcription factor and RNA polymerase binding energies with a comprehensive thermodynamic model of gene regulation. The model was validated by predicting and then measuring the variability of lac operon regulation in a collection of natural isolates. We then implement the model to analyze the sensitivity of the promoter sequence to the regulatory output, and predict the potential for regulation to evolve due to point mutations promoter region.

\section{Keywords}

thermodynamic models; lac operon; evolutionary potential; transcriptional regulation; natural variability

\section{Introduction}

Despite efforts to understand genotypic variability within natural populations [1] and recent interest in fine-tuning genetic circuits for synthetic biology [2], it still remains unclear how, 
with base pair resolution, the sequence of a gene regulatory region can be translated into output levels of gene expression [3]. Generally, classical population genetics has treated regulatory architectures as changeless parameters, rather than potential evolutionary variables, focusing on changes in protein structure rather than gene regulation. However, genetic regulatory architecture can also determine the variation of traits, and thus the evolutionary potential of these genes [4]. After all, the structure of bacterial promoters dictates interactions among the transcriptional apparatus, and through the modification of this structure, regulatory circuits can be modified to potentially allow cells to occupy different niches $[5,6]$.

Thermodynamic models of gene regulation have been widely used as a theoretical framework to dissect and understand genetic architectures [7, 8, 9, 10,11]. Such dissections have led to a quantitative understanding of how parameters such as binding energies, transcription factor copy numbers, and the mechanical properties of the DNA dictate expression levels. Recently the development of experimental techniques combining these types of models with cell sorting and high-throughput sequencing have made it possible to understand gene regulation at single-base pair resolution [12,13,14], as well as to deliberately design promoter architectures with desired input-output functions [15]. These models connect the sequence of a promoter to the output phenotype, making it possible to predict variability and evolutionary potential of gene regulatory circuits.

The lac operon has served as a paradigm of a genetic regulatory system for more than 60 years $[16,17]$. This operon contains the molecular machinery that some bacterial species, including the model organism E. coli use to import and consume lactose. Extensive quantitative characterization of the regulation of this genetic circuit $[18,19]$, as well as of the link between fitness and expression of the operon [20,21, 22, 23, 24] make it an ideal system for exploring the evolutionary potential of a regulatory circuit. With previous exhaustive description and quantification of the parameters controlling the expression level of this genetic circuit $[19,25,26,27]$ we now have what we think is a nearly complete picture of the regulatory knobs that can modify the expression level, shown schematically in Figure 1(a). In this article we build upon this understanding by directly linking the sequence of the promoter region with these control parameters, thereby creating a map from genotype to transcriptional output.

Within a collection of $E$. coli isolated from different host organisms we observe significant variability for the regulation of the lac operon, as shown in Figure 1(b). By characterizing the variability of the regulatory control parameters shown in Figure 1(a) within these strains, we identified evolutionary trends in which certain parameters or subsets of parameters are seen to vary more often than others within this collection of natural isolates. Using the map of promoter sequence to transcriptional output, we demonstrated that the regulatory inputoutput function for the lac promoter could account for most of the natural variability in regulation we observed. We then implement the map to explore the theoretical potential for this regulatory region to evolve. This level of analysis gives us clues as to how selection could fine tune gene expression levels according to the environmental conditions to which cells are exposed. 


\section{Results}

\subsection{Quantitative model of the natural parameters that regulate gene expression}

Thermodynamic models of gene regulation have become a widely used theoretical tool to understand and dissect different regulatory architectures [3, 12, 19, 26, 27, 31]. The lac promoter is one such regulatory architecture that has been studied in detail [32]. Models have been constructed and experimentally validated for both the wild-type lac promoter and synthetic promoter regions built up from the lac operon's regulatory components $[12,15$, $19,26,27,32,33,34,35,36,37]$

In a simple dynamical model of transcription the number of messenger RNA (mRNA) is proportional to the transcription rate and the degradation rate of the mRNA,

$$
\frac{d m}{d t}=-\gamma \cdot m+\sum_{i} r_{i} \cdot p_{i}
$$

where $\gamma$ is the mRNA degradation rate and $m$ is the number of transcripts of the gene per cell; $r_{i}$ and $p_{i}$ are the transcription rate and the probability of state $i$ respectively. We can think of $p_{i}$ as a measure of the time spent in the different transcriptionally active states. Thermodynamic models assume that the gene expression level is dictated by the probability of finding the RNA polymerase (RNAP) bound to the promoter region of interest [7, 8, 9]. With a further quasi-equilibrium assumption for the relevant processes leading to transcription initiation, we derive a statistical mechanics description of how parameters such as transcription factor copy number and their relevant binding energies, encoded in the DNA binding site sequence, affect this probability [10]. Quantitative experimental tests of predictions derived from equilibrium models have suggested the reasonableness of the assumption [15, 19, 26, 27], although caution should be used as the equilibrium assumption is not necessarily valid in all cases. The validity of this equilibrium assumption relies on the different time-scales of the processes involved in the transcription of a gene. Specifically the rate of binding and unbinding of the transcription factors and the RNAP from the promoter region should be faster than the open complex formation rate; if so, the probability of finding the RNAP bound to the promoter is given by its equilibrium value $[9,38]$. For the case of the Lac repressor, the rate of unbinding from the operator is $0.0221 / \mathrm{s}$ [39], and the binding of an unoccupied operator with 10 repressors per cell occurs at a similar rate [40]. Open complex formation, a rate limiting step in promoter escape, has been measured at a rate of $2 \times 10^{-3} 1 / \mathrm{s}$ [41]. Promoter escape is about an order to magnitude slower than the binding and unbinding of the Lac repressor, and this separation of time scales supports the equilibrium assumption for this particular case. We enumerate the possible states of the system and assign statistical weights according to the Boltzmann distribution as shown in Figure 2 .

From these states and weights we derive an equation describing the probability of finding the system in a transcriptionally active state, and therefore the production term from Equation 1, 


$$
\sum_{i} r_{i} p_{i}=\sum_{i} r_{i} \frac{W_{i}}{Z_{t o t}}
$$

where $W_{i}$ is the statistical weight of states in which the polymerase is bound, which are assumed to lead to the transcription of the operon (shaded blue in Figure 2), and $Z_{t o t}=$ $\Sigma_{\text {All states }} W_{\text {state }}$ is the partition function, or the sum of the statistical weights of all states. We connect this model to experimental measurements of repression, that is the ratio of gene expression in the absence of the active repressor to gene expression in the presence of active repressor, using:

$$
\text { repression }=\frac{\text { gene } \operatorname{expression}(R=0)}{\text { gene } \operatorname{expression}(R \neq 0)}
$$

where $R$ is the number of repressor molecules per cell. The experimental equivalent of repression is depicted in Figure 1(c). In experiments, IPTG is used to inactivate the Lac repressor, preventing it from binding to the genome with high affinity [19]. Repression, as defined in Equation 3, has been a standard metric for the role of transcription factors, including the Lac repressor, on gene expression [7, 42]. By measuring the ratio of steadystate levels of a gene reporter protein, here LacZ, we are able to isolate the role of the repressor in gene regulation, as described further in section S8 of the Supplemental Material.

Various models of the WT lac promoter have been reported in the past using this simple structure. Our work builds upon the work by Kinney et al. [12]. Kinney and collaborators combined a thermodynamic model of regulation with high-throughput sequencing to predict gene expression from statistical sequence information of the CRP and the RNAP binding sites. To predict how the sequence of the entire regulatory region influences expression, we adapted this model to account for how the binding site sequence and copy number of the Lac repressor modulate gene expression. Our model also takes into account growth rate effects, captured in the RNAP copy number [43, 44].

Based on previous work done on the lac operon [19, 12], we assumed that the presence of the activator doesn't affect the rate of transcription ( $r_{i}$ from Equation 1$)$, but the probability of recruiting the polymerase to the promoter ( $p_{i}$ from Equation 1). Previous experimental characterization of the repressor binding energy to the different operators [26], the looping free energy for the upstream loop between $O_{1}-O_{3}$ [27], activator concentration and its interaction energy with RNAP [19], RNAP binding energy [15] and RNAP copy number as a function of the growth rate [44], left us only with three unknown parameters for the model. One of these missing parameters, a decrease in the looping free energy when CRP and Lac repressor are bound at the same time, is a consequence of the experimental observation that the presence of CRP stabilizes the formation of the loop between $O_{1}-O_{3}[29,45]$. The remaining two parameters, the looping energies for the $\mathrm{O}_{1}-\mathrm{O}_{2}$ and $\mathrm{O}_{3}-\mathrm{O}_{2}$ loops are not well characterized. These looping energies may differ from upstream loops due to the absence of the RNAP binding site which modifies the mechanical properties of the loop [46]. We fit these parameters for our model using Oehler et al. repression measurements on lac operon constructs with partially mutagenized or swapped binding sites [42,47] (see 
section S5 of the Supplemental Material for further details). Using these parameters the model is consistent with previous measurements (Figure S4). We emphasize that having the 14 parameters of the model characterized (see Table $\mathrm{S} 1$ ) provides testable predictions without free parameters that we compare with our experimental results.

\subsection{Sensitivity of expression to model parameters}

As an exploratory tool, the model can predict the change in regulation due to modifications in the promoter architecture. Figure 3 shows the fold-change in the repression level as a function of each of the parameters, using the lab strain MG1655 as a reference state (see Supplemental Material for further detail on these reference parameters). We have reported parameters using strain MG1655 as a reference strain because this strain served as the basis for which most parameter values were determined and the gene expression model was derived.

From this figure we see that within the confines of this model, modifications in the $O_{1}$ binding energy have the most drastic effect on the repression of the operon. For the case of $\mathrm{O}_{2}$ we see that increasing its affinity for the repressor does not translate into an increased ability to turn off the operon; but by decreasing this operator affinity the model predicts a reduction in the repression with respect to the reference strain.

Surprisingly the repression level is predicted to be insensitive to activator copy number. The same cannot be said about the affinity of the activator, since decreasing the activator binding energy greatly influences the repression level.

\subsection{Mapping from sequence space to level of regulation}

Recent developments of an experimental technique called sort-seq, involving cell sorting and high-throughput sequencing, have proved to be very successful in revealing how regulatory information is encoded in the genome with base pair resolution [12]. This technique generates energy matrices that make it possible to map from a given binding site sequence to its corresponding binding energy for a collection of different proteins and binding sites. Combining these energy matrices with thermodynamic models enables us to convert promoter sequence to the output level of gene expression. Recently these energy matrices have been used to deliberately design promoters with a desired expression level, demonstrating the validity of these matrices as a design tool for synthetic constructs [15]. We use the matrices for CRP and RNAP published previously [12]. We experimentally determined the matrix for the LacI operator using previously published methods [12], as discussed in Materials and Methods. Figure 4(a) shows a schematic representation of the relevant protein binding sites involved in the regulation of the lac operon and their respective energy matrices. Implementing these matrices into the thermodynamic model gives us a map from genotype to phenotype. We use this map to calculate the fold-change in repression relative to MG1655 for all possible point mutations in this region. Figure 4(b) shows the fold-changes in repression levels for the two base pair substitutions at each position that result in the largest predicted increase or decrease in repression. 
Again we see that mutations in the $O_{1}$ binding site have the largest effect on regulation since a single base pair change can lower the ability of the cell to repress the operon by a factor of $\approx 20$. With only two relevant mutation that could significantly increase the repression level, this map reveals how this operator and its corresponding transcription factor diverged in a coordinated fashion; the WT sequence has nearly maximum affinity for the repressor [48]. It is known that the non-natural operator $O_{i d}$ binds more strongly than $O_{1}$ [42]. $O_{i d}$ is one base pair shorter than $O_{1}$ and current maps made with sort-seq cannot predict changes in binding affinity for binding sites of differing length, although accounting for length differences in binding sites is not a fundamental limitation of this method.

For the auxiliary binding sites, the effect discussed in section 2.2 is reflected in this map: increasing the Lac repressor affinity for the $\mathrm{O}_{2}$ binding site does not increase repression. Mutations in almost all positions can decrease repression, and no base pair substitutions significantly increase the repression level. Mutations in the $O_{3}$ binding site have the potential to either increase or decrease the repression level. With respect to the RNAP binding site, we can see that, as expected, the most influential base pairs surround the well characterized -35 and -10 boxes. The CRP binding site overlaps three base pairs with the upstream Lac repressor auxiliary operator. As the heat-map reveals, the binding energy is relatively insensitive to changes in those base pairs, so we assume independence when calculating the binding energy and capture the synergy between the Lac repressor bound to $\mathrm{O}_{3}$ and CRP with an interaction energy term.

The construction of the sequence to phenotype map enables us to predict the evolvability of our promoter region. We calculated the effect that all possible double mutations would have in the regulation of the operon, again with respect to the predicted repression level of the reference strain MG1655. Figure 5 shows what we call the "phenotype change distribution" obtained by mutating one or two base pairs from the reference sequence, under the assumption of same growth rate and transcription factor copy numbers as the reference strain. The distribution peaks at zero for both cases, meaning that the majority of mutations are predicted not to change the repression level with respect to the reference strain, and would result in genetic drift. However it is interesting to note that the range of repression values predicted by the model with only one mutation varied between 30 times lower and 4.6 times higher than the reference value, and with two mutations the repression varied between 345 times lower and 15 times higher than the reference value. This suggests that regulation of this operon could rapidly adapt and fine tune regulation given appropriate selection.

\subsection{Promoter sequence variability of natural isolates and available sequenced genomes}

In order to explore the natural variability of this regulatory circuit, we analyzed the lac promoter region of 22 wild-type E. coli strains which were isolated from different organisms [30], along with 69 fully sequenced E. coli strains (including MG1655) available online (http://www.ncbi.nlm.nih.gov/genomes/MICROBES/microbial_taxtree.html). Figure 6 summarizes the sequencing results; for comparison, we plot the "genotype to phenotype map" from Figure 4(b) to gain insight into how the sequence variability influences regulation in these strains. Figure 6(b) shows the relative frequency of single nucleotide 
polymorphisms (SNP's) with respect to the consensus sequence. Qualitatively we can appreciate that the mutations found in these strains fell mostly within base pairs which according to the model weakly regulated expression. To quantify this observation we mapped the sequences to their corresponding binding energies. As shown in Figure 6(c) the distribution of parameters is such that the observed mutations result in relatively small changes to the binding energies, less than $1 k_{B} T$ relative to the reference sequence, except for the $O_{3}$ binding energy that is predicted to increase $>1 k_{B} T$ in 16 strains.

\subsection{Does the model account for variability in the natural isolates?}

Next we further characterized the eight strains from Figure 1(b) in order to determine if the observed variability in regulation could be accounted for in the model (see section S2 for details on the $16 \mathrm{~S}$ rRNA of this subset of strains). In particular, we measured the in vivo repressor copy number with quantitative immunoblots (see Material and Methods) and the growth rate. Table 1 shows the measured repressor copy number and the doubling time for these strains.

Using the thermodynamic model by taking into account the repressor copy number, the promoter sequence and the growth rate, we predict the repression level for each of the isolates measured in Figure 1(b). In Figure 7 we plot these predicted values vs. the experimental measurements. We find that the model accounts for the overall trends observed in the isolates, with the predictions for 6 of 8 strains falling within two standard deviations of the measurements. A few of the measured repression values fall outside of the prediction, suggesting that the model may not capture the full set of control parameters operating in all of the strains.

\subsection{Exploring the variability among different species}

We extended our analysis to different microbial species with similar lac promoter architectures. After identifying bacterial species containing the lac repressor, we used the sort-seq derived energy matrices shown in Figure 4(a) to identify the positions of the transcription factor binding sites in each of these candidate strains. We identified a set of eight species whose lac promoter architecture was similar to E. coli. Figure 8 shows the $16 \mathrm{~S}$ rRNA phylogenetic tree for these strains. The predicted change in regulation was calculated for these strains using the model whose states are shown in Figure 2, the energy matrices in Figure 4(a), and assuming all strains have the same growth rate and transcription factor copy numbers as the lab strain MG1655. The repression level relative to $E$. coli among these species is predicted to increase as much as a factor of $\approx 20$ and decrease as much as a factor of $\approx 4$. Regulation of the operon seems to follow phylogenetic patterns in the 16S rRNA tree, with E. coli relatives having a similar predicted repression level, Citrobacter evolved to increase repression, and Salmonella evolved to decrease repression.

\section{Discussion}

The approach presented here combines thermodynamic models of gene regulation with energy matrices generated with sort-seq to produce a single-base pair resolution picture of the role that each position of the promoter region has in regulation. These types of models

Phys Biol. Author manuscript; available in PMC 2015 April 01. 
based on equilibrium statistical mechanics have been used previously for the lac operon [19, 25], here we expanded the model to account for important cellular parameters such as growth rate, the binding site strengths of all transcription factors, and the binding site strength of RNAP. Thermodynamic models are functions of the natural variables of the system as opposed to the widely used phenomenological Hill functions [49], where it is less straightforward how changes to a promoter region translate to changes in regulatory parameters such as $K_{M}$, the half saturation constant, and $n$, the Hill coefficient. Currently our model assumes that protein-protein interactions and DNA looping energies are kept constant, but these variables could also be a function of the promoter sequence, affecting the positioning of the transcription factors and therefore their interactions with the other molecules involved.

The underlying framework developed here can be applied to any type of architecture. Here we use the lac operon because it is well characterized. There is no reason to believe that this approach could not be extended to other regulatory regions, however such an effort would require extensive quantitative characterization of the control parameters of each genetic circuit, such as protein copy numbers, interaction energies, and binding affinities. Although this level of characterization requires additional experimental effort, we believe that developing such predictive, single-base pair models of gene regulation can lead to significant insights into the how genetic circuits function, interact with each other, and evolve.

The majority of the natural variability found among the sequenced promoters tended to fall in bases predicted to have low impact on overall regulation, as shown in Figure 6. As an example the highly conserved mutation in the CRP binding energy or the mutations along the RNAP binding site are predicted to change the binding energy by less than $1 k_{B} T$, having a very low impact on the repression level. With respect to the repressor binding sites, amongst the sequenced natural isolates only one mutation was found in the $\mathrm{O}_{2}$ binding site. Unlike the $O_{1}$ and $O_{3}$ operators, the evolution of $O_{2}$ may be constrained given that its sequence encodes both gene regulatory information and is part of the coding region of the $\beta$ galactosidase gene.

As shown in Figure 7, after taking into account the variability in the promoter sequence, changes in the repressor copy number, and changes in the growth rate the model accounts for most of the variability in regulation for the majority of the isolates. Linear regression of the entire experimental dataset weighted by the inverse of their standard deviation gives a slope of 1.26 with an $R^{2}$ of 0.24 . It can be seen that many of the points fall close to or on the $\mathrm{x}=\mathrm{y}$ line, indicating that the poor fit is a result of a few outliers within the dataset. Removing the outliers (Perching bird, Human-MA, and Human-NY) results in a best fit line of slope 1.05 with $R^{2} 0.74$, reiterating that the model is consistent with the phenotype of 5 of 8 isolates. It is interesting that the three isolates whose regulatory outputs were predicted poorly by the model (Perching bird, Human-MA, and Human-NY in Figure 7) all have identical promoter sequences, which is the consensus promoter sequence as shown in Figure S1. Although these three strains have identical sequences, two strains repressed more than predicted and the other strain repressed less. This indicates there are likely other cellular parameters that influence gene expression levels that are not included in the model. 
Currently the model cannot take into account variation in the protein structure of the transcription factors or the RNAP and its sigma factors. Changes in these proteins could account for some of the discrepancies between the model and the observed levels of regulation. It is likely that some global parameters that modulate transcriptional outputs which are not accounted for in the model also contribute to the disagreement with model predictions. We note that repression is a measurement of expression relative to expression in the absence of the repressor. This definition enables us to isolate the role of a particular transcription factor in regulation. Therefore, as discussed in section S8, some global regulatory parameters such as ribosomal binding sites of the relevant genes and variables such as the ribosome copy number should not impact repression levels.

From an evolutionary perspective, it is interesting that the regulation seems to be more sensitive to changes in the activator binding energy than to the activator protein copy number, as shown in Figure 3. This result might be attributed to the nature of this transcription factor. CRP is known to be a "global" transcription factor that regulates $>50 \%$ of the E. coli transcription units [50]. Given its important global role in the structure of the transcriptome, changing the copy number of CRP would have a global impact on expression whereas tuning its binding affinity at a particular regulatory region has a local impact on one promoter. The regulatory knob of CRP copy number not influencing expression at the lac operon indicates this regulatory region may have evolved to be robust against changes in this global regulatory parameter.

The fact that the $O_{3}$ operator has the possibility to change in both directions (greater or lower affinity) as reflected in Figure 4(b) suggests plasticity of the operon, allowing it to evolve according to environmental conditions. In fact this parameter changed the most among the related microbial species as shown in Figure 8(b), having species such as Citrobacter koser $i$ with an operator predicted to be $5 k_{B} T$ stronger than the reference value, and other species such as Salmonella bongori that completely lost this binding site. Although we don't yet know whether these regulatory predictions will be borne out in experimental measurements, this analysis demonstrates the utility of our sequence-tophenotype map in interpreting the consequences of variability within the regulatory regions of sequenced genomes.

To the best of our knowledge Figure 5 shows the first quantification of how easily regulation can change given one or two point mutations along the entire promoter region. Previous studies were limited to a subset of base pairs in the Lac repressor operators and two amino acid substitutions in the Lac repressor [51]. The distribution of predicted phenotypes is very sharp close to the reference value, as a consequence the majority of the possible mutations would not be selected on. But given that regulation can change by an order of magnitude or more in both directions (increased or decreased repression) with only two mutations, changing the regulatory region of the gene could function as a fast response strategy of adaptation.

It is known from previous work that lac operon expression can have an impact on cell fitness [20, 21, 22, 24]. Under laboratory conditions, high expression of the lac operon resulted in loss of fitness due to expression of $\operatorname{lac} Y$, a transporter which imports lactose into 
the cell. This would suggest regulation is essential to avoid the negative consequences of lacY overexpression, and tight regulation would be selected. However it is possible that natural selection would act also to modulate the magnitude of the response. Strains exposed to environments with periodical bursts of lactose could trigger instantly a high gene dosage, resulting in a steeper slope on an induction curve, while strains rarely exposed to lactose would have a moderate response, i.e. a less steep induction curve. Our exploration and prediction of regulatory phenotypes in sequenced genomes shows that the biggest changes in regulation were found to increase repression (see Supplemental Material), suggesting that lactose might not be present regularly in the natural environment of some strains.

The combination of thermodynamic models with sort-seq generated energy matrices presented here promises to be an useful tool to study the evolution of gene regulation. This theoretical framework allows us to explore the effect that the modification of control parameters can have in the expression levels, and predict how point mutations in gene promoter regions enable cells to evolve their gene regulatory circuits.

\section{Materials and methods}

\subsection{Growth conditions}

Unless otherwise indicated, all experiments started by inoculating the strains from frozen stocks kept at $-80^{\circ} \mathrm{C}$. Cultures were grown overnight in Luria Broth (EMD, Gibbstown, NJ) at $37^{\circ} \mathrm{C}$ with shaking at $250 \mathrm{rpm}$. In all of the experiments these cultures were used to inoculate three replicates for each of the relevant conditions, diluting them 1:3000 into $3 \mathrm{~mL}$ of M9 buffer ( $2 \mathrm{mM} \mathrm{MgSO}_{4}, 0.10 \mathrm{mM} \mathrm{CaCl} 2,48 \mathrm{mM} \mathrm{Na}_{2} \mathrm{HPO}_{4}, 22 \mathrm{mM} \mathrm{KH}_{2} \mathrm{PO}_{4}, 8.6 \mathrm{mM}$ $\mathrm{NaCl}, 19 \mathrm{mM} \mathrm{NH}_{4} \mathrm{Cl}$ ) with $0.5 \%$ glucose and $0.2 \%$ casamino acids (here referred to as "supplemented M9"). Cells were cultured at $37^{\circ} \mathrm{C}$ with shaking at $250 \mathrm{rpm}$ and harvested at the indicated $O D_{600}$.

\subsection{Gene expression measurements}

To perform the LacZ assay we followed the protocol used by Garcia and Phillips [26]. Strains were grown in supplemented $\mathrm{M} 9$ for approximately 10 generations and harvested at an $O D_{600}$ around 0.4. A volume of the cells was added to Z-buffer ( $60 \mathrm{mM} \mathrm{Na}_{2} \mathrm{HPO}_{4}, 40$ $\mathrm{mM} \mathrm{NaH} \mathrm{PO}_{4}, 10 \mathrm{mM} \mathrm{KCl}, 1 \mathrm{mM} \mathrm{MgSO} \mathrm{S}_{4}, 50 \mathrm{mM} \beta$-mercaptoethanol, $\mathrm{pH}$ 7.0) for a total volume of $1 \mathrm{~mL}$. For fully induced cells we used $50 \mu \mathrm{L}$ and for uninduced cultures we concentrated the cells by spinning down $1 \mathrm{~mL}$ of culture and resuspending in Z-buffer. The cells were lysed by adding $25 \mu \mathrm{L}$ of $0.1 \%$ SDS and $50 \mu \mathrm{L}$ of chloroform and vortexing for 15 seconds. To obtain the readout, we added $200 \mu \mathrm{L}$ of $4 \mathrm{mg} / \mathrm{mL} 2$-nitrophenyl $\beta$-Dgalactopiranoside (ONPG). Once the solution became noticeably yellow, we stopped the reaction by adding $200 \mu \mathrm{L}$ of $2.5 \mathrm{M} \mathrm{Na}_{2} \mathrm{CO}_{3}$.

To remove cell debris we spun down the tubes at $13000 \times \mathrm{g}$ for 3 minutes. $200 \mu \mathrm{L}$ of the supernatant were read at $O D_{420}$ and $O D_{550}$ on a microplate reader (Tecan Safire2). The absolute activity of LacZ was measured in Miller units as 


$$
M U=1000 \times \frac{O D_{420}-1.75 \times O D_{550}}{t \times v \times O D_{600}} \times 0.826
$$

where $t$ is the time we let the reaction run and $v$ is the volume of cells used in $\mathrm{mL}$. The factor of 0.826 adjusts for the concentration of ONP relative to the standard LacZ assay.

\subsection{Measuring in-vivo lac repressor copy number}

To measure the repressor copy number of the natural isolates we followed the same procedure reported by Garcia and Phillips [26]. Strains were grown in $3 \mathrm{~mL}$ of supplemented M9 until they reached an $O D_{600} \approx 0: 4-0.6$. Then they were transferred into $47 \mathrm{~mL}$ of warm media and grown at $37^{\circ} \mathrm{C}$ to an $O D_{600}$ of $0.4-0.6 .45 \mathrm{~mL}$ of culture were spun down at $6000 \times \mathrm{g}$ and resuspended into $900 \mu \mathrm{L}$ of breaking buffer $(0.2 \mathrm{M}$ Tris- $\mathrm{HCl}, 0.2$ $\mathrm{M} \mathrm{KCl}, 0.01 \mathrm{M}$ Magnesium acetate, $5 \%$ glucose, $0.3 \mathrm{mM}$ DTT, $50 \mathrm{mg} / 100 \mathrm{~mL}$ lysozyme, 50 $\mu \mathrm{g} / \mathrm{L}$ phenylmethanesulfonyluoride (PMSF), $\mathrm{pH}$ 7.6).

Cells were lysed by performing four freeze-thaw cycles, adding $4 \mu \mathrm{L}$ of a 2,000 Ku-nitz/mL DNase solution and $40 \mu \mathrm{L}$ of a $1 \mathrm{M} \mathrm{MgCl}$ solution and incubating at $4{ }^{\circ} \mathrm{C}$ with mixing for 4 hours after the first cycle. After the final cycle, cells were spun down at 13,000 $\times \mathrm{g}$ for $45 \mathrm{~min}$ at $4{ }^{\circ} \mathrm{C}$. We then obtained the supernatant and measured its volume. The pellet was resuspended in $900 \mu \mathrm{L}$ of breaking buffer and again spun down at $15,000 \times \mathrm{g}$ for $45 \mathrm{~min}$ at $4^{\circ} \mathrm{C}$. In order to review the quality of the lysing process, $2 \mu \mathrm{L}$ of this resuspended pellet was used as a control to ensure the luminescent signal of the resuspension was $<30 \%$ of the sample.

To perform the immuno-blot we pre-wet a nitrocellulose membrane $(0.2 \mu \mathrm{M}$, Bio-Rad $)$ in TBS buffer (20 mM Tris $-\mathrm{HCl}, 500 \mathrm{mM} \mathrm{NaCl})$ and left it to air dry. For the standard curve a purified stock of Lac repressor tetramer [46] was serially diluted into HG105 ( $\Delta$ lacI strain) lysate. $2 \mu \mathrm{L}$ were spotted for each of the references and each of the samples. After the samples were visibly dried the membrane was blocked using TBST (20 mM Tris Base, 140 $\mathrm{mM} \mathrm{NaCl}, 0.1 \%$ Tween $20, \mathrm{pH} 7.6)+2 \% \mathrm{BSA}+5 \%$ dry milk for $1 \mathrm{~h}$ at room temperature with mixing. We then incubated the membrane in a 1:1000 dilution of anti-LacI monoclonal antibody (from mouse; Millipore) in blocking solution for $1.5 \mathrm{~h}$ at room temperature with mixing. The membrane was gently washed with $\mathrm{TBS} \approx 5$ times. To obtain the luminescent signal the membrane was incubated in a 1:2000 dilution of HRP-linked anti-mouse secondary antibody (GE Healthcare) for $1.5 \mathrm{~h}$ at room temperature with mixing and washed again $\approx 5$ times with TBS. The membrane was dried and developed with Thermo Scientific Super-Signal West Femto Substrate and imaged in a Bio-Rad VersaDoc 3000 system.

\subsection{Constructing the in-vivo lac repressor energy matrix}

The energy matrix was inferred from sort-seq data in a manner analogous to methods described in Kinney PNAS 2010 [12]. Briefly, a library of mutant lac promoters was constructed in which the region [-100:25] (where coordinates are with respect to the transcription start site) was mutagenized with a $3 \%$ mutation rate. The transcriptional activity of each mutant promoter was measured by flow cytometry using a GFP reporter. To fit the LacI energy matrix, we used a Markov chain Monte Carlo algorithm to fit an energy 
matrix to the LacI $O_{1}$ binding site by maximizing the mutual information between energies predicted by the matrix and flow cytometry measurements. The justification for maximizing mutual information is described in detail in [12, 52].

\section{Supplementary Material}

Refer to Web version on PubMed Central for supplementary material.

\section{Acknowledgments}

We would like to acknowledge Ron Milo, Niv Antonovsky, Adrian Jinich, Sushant Sundaresh, Joanna Robaszewski and Hernan Garcia for useful discussions. We are grateful to Valeria Souza (UNAM) for her kind donation of the $E$. coli strains. This work was supported by the National Institutes of Health, grant numbers DP1 OD000217A (Directors Pioneer Award), R01 GM085286 and R01 GM085286B (www.nih.gov). This work was also supported by the Donna and Benjamin M. Rosen Center for Bioengineering at Caltech. The funders had no role in study design, data collection and analysis, decision to publish, or preparation of the manuscript.

\section{References}

1. Thompson JR, Pacocha S, Pharino C, Klepac-Ceraj V, Hunt DE, Benoit J, Sarma-Rupavtarm R, Distel DL, Polz MF. Genotypic diversity within a natural coastal bacterioplankton population. Science (New York, N.Y.). 2005; 307(5713):1311-1313.

2. Zelcbuch L, Antonovsky N, Bar-Even A, Levin-Karp A, Barenholz U, Dayagi M, Liebermeister W, Flamholz A, Noor E, Amram S, Brandis A, Bareia T, Yofe I, Jubran H, Milo R. Spanning highdimensional expression space using ribosome-binding site combinatorics. Nucleic acids research. 2013; 41(9):e98. [PubMed: 23470993]

3. Segal E, Widom J. From DNA sequence to transcriptional behaviour: a quantitative approach. Nature reviews. Genetics. 2009; 10(7):443-456.

4. Hansen TE. The Evolution of Genetic Architecture. Annual Reviews of Ecology Evolution, and Systtematics. 2006 May.37:123-157.

5. McAdams HH, Srinivasan B, Arkin AP. The evolution of genetic regulatory systems in bacteria. Nature reviews. Genetics. 2004; 5(3):169-178.

6. Perez JC, Groisman Ea. Evolution of transcriptional regulatory circuits in bacteria. Cell. 2009; 138(2):233-244. [PubMed: 19632175]

7. Ackers GK, Johnson AD, Shea AM. Quantitative model for gene regulation by lambda phage repressor. Proceedings of the National Academy of Sciences of the United States of America. 1982; 79(4):1129-1133. [PubMed: 6461856]

8. Buchler NE, Gerland U, Hwa T. On schemes of combinatorial transcription logic. Proceedings of the National Academy of Sciences of the United States of America. 2003; 100(9):5136-5141. [PubMed: 12702751]

9. Bintu L, Buchler NE, Garcia HG, Gerland U, Hwa T, Kondev J, Phillips R. Transcriptional regulation by the numbers: models. Current opinion in genetics \& development. 2005; 15(2):116124. [PubMed: 15797194]

10. Bintu L, Buchler NE, Garcia HG, Gerland U, Hwa T, Kondev J, Kuhlman T, Phillips R. Transcriptional regulation by the numbers: applications. Current opinion in genetics \& development. 2005; 15(2):125-135. [PubMed: 15797195]

11. Sherman MS, Cohen BA. Thermodynamic state ensemble models of cis-regulation. PLoS computational biology. 2012; 8(3):e1002407. [PubMed: 22479169]

12. Kinney JB, Murugan A, Callan CG, Cox EC. Using deep sequencing to characterize the biophysical mechanism of a transcriptional regulatory sequence. PNAS. 2010; 107(20):91589163. [PubMed: 20439748]

13. Sharon E, Kalma Y, Sharp A, Raveh-Sadka T, Levo M, Zeevi D, Keren L, Yakhini Z, Weinberger A, Segal E. Inferring gene regulatory logic from high-throughput measurements of thousands of systematically designed promoters. Nature biotechnology. 2012; 30(6):521-530. 
14. Melnikov A, Murugan A, Zhang X, Tesileanu T, Wang L, Rogov P, Feizi S, Gnirke A, Callan CG, Kinney JB, Kellis M, Lander ES, Mikkelsen TS. Systematic dissection and optimization of inducible enhancers in human cells using a massively parallel reporter assay. Nature biotechnology. 2012; 30(3):271-277.

15. Brewster RC, Jones DL, Phillips R. Tuning Promoter Strength through RNA Polymerase Binding Site Design in Escherichia coli. PLoS Computational Biology. 2012; 8(12):e1002811. [PubMed: 23271961]

16. Wilson CJ, Zhan H, Swint-Kruse L, Matthews KS. The lactose repressor system: paradigms for regulation, allosteric behavior and protein folding. Cellular and molecular life sciences : CMLS. 2007; 64(1):3-16. [PubMed: 17103112]

17. Reznikoff WS. The lactose operon-controlling elements: a complex paradigm. Molecular microbiology. 1992; 6(17):2419-2422. [PubMed: 1328815]

18. Setty Y, Mayo AE, Surette MG, Alon U. Detailed map of a cis-regulatory input function. Proceedings of the National Academy of Sciences of the United States of America. 2003; 100(13): 7702-7707. [PubMed: 12805558]

19. Kuhlman T, Zhang Z, Saier MH, Hwa T. Combinatorial transcriptional control of the lactose operon of Escherichia coli. Proceedings of the National Academy of Sciences of the United States of America. 2007; 104(14):6043-6048. [PubMed: 17376875]

20. Dean AM. Selection and Neutrality in Lactose Operons of Escherichia coli. Genetics. 1989; 123:441-454. [PubMed: 2513251]

21. Dekel E, Alon U. Optimality and evolutionary tuning of the expression level of a protein. Nature. 2005; 436(7050):588-592. [PubMed: 16049495]

22. Perfeito L, Ghozzi S, Berg J, Schnetz K, Lässig M. Nonlinear Fitness Landscape of a Molecular Pathway. PLoS genetics. 2011; 7(7):1-10.

23. Poelwijk FJ, Heyning PD, de Vos MGJ, Kiviet DJ, Tans SJ. Optimality and evolution of transcriptionally regulated gene expression. BMC systems biology. 2011; 5(1):128. [PubMed: 21846366]

24. Eames M, Kortemme T. Cost-benefit tradeoffs in engineered lac operons. Science (New York, N.Y.). 2012; 336(6083):911-915.

25. Vilar JMG. Accurate prediction of gene expression by integration of DNA sequence statistics with detailed modeling of transcription regulation. Biophysical journal. 2010; 99(8):2408-2413. [PubMed: 20959080]

26. Garcia HG, Phillips R. Quantitative dissection of the simple repression input-output function. Proceedings of the National Academy of Sciences of the United States of America. 2011; 108(29): 12173-12178. [PubMed: 21730194]

27. Boedicker JQ, Garcia HG, Phillips R. Theoretical and Experimental Dissection of DNA LoopMediated Repression. Physical Review Letters. 2013; 110(1):018101. [PubMed: 23383841]

28. Tagami H, Aiba H. Role of CRP in transcription activation at Escherichia coli lac promoter: CRP is dispensable after the formation of open complex. Nucleic acids research. 1995; 23(4):599-605. [PubMed: 7899079]

29. Hudson JM, Fried MG. Co-operative interactions between the catabolite gene activator protein and the lac repressor at the lactose promoter. Journal of molecular biology. 1990; 214(2):381-396. [PubMed: 2166165]

30. Souza V, Rocha M, Valera A, Luis E, Eguiarte LE. Genetic Structure of Natural Populations of Escherichia coli in Wild Hosts on Different Continents. Applied and Environmental Microbiology. 1999; 65(8):3373-3385. [PubMed: 10427022]

31. Segal E, Raveh-Sadka T, Schroeder M, Unnerstall U, Gaul U. Predicting expression patterns from regulatory sequence in Drosophila segmentation. Nature. 2008; 451(7178):535-540. [PubMed: 18172436]

32. Saiz L, Vilar JMG. Ab initio thermodynamic modeling of distal multisite transcription regulation. Nucleic acids research. 2008; 36(3):726-731. [PubMed: 18056082]

33. Vilar JMG, Leibler S. DNA Looping and Physical Constraints on Transcription Regulation. Journal of Molecular Biology. 2003; 331(5):981-989. [PubMed: 12927535] 
34. Vilar JMG, Saiz L. DNA looping in gene regulation: from the assembly of macromolecular complexes to the control of transcriptional noise. Current opinion in genetics \& development. 2005; 15(2):136-144. [PubMed: 15797196]

35. Saiz L, Vilar JMG. Multilevel deconstruction of the In vivo behavior of looped DNA-protein complexes. PloS one. 2007; 2(4):e355. [PubMed: 17406679]

36. Saiz L, Vilar JMG. DNA looping: the consequences and its control. Current opinion in structural biology. 2006; 16(3):344-350. [PubMed: 16714105]

37. Saiz L, Rubi JM, Vilar JMG. Inferring the in vivo looping properties of DNA. Proceedings of the National Academy of Sciences of the United States of America. 2005; 102(49):17642-17645. [PubMed: 16303869]

38. Rydenfelt M, Cox R, Garcia H, Phillips R. Statistical mechanical model of coupled transcription from multiple promoters due to transcription factor titration. Physical Review E. 2014; 89(1): 012702.

39. Nelson HC, Sauer RT. Lambda repressor mutations that increase the affinity and specificity of operator binding. Cell. 1985; 42(2):549-558. [PubMed: 3161621]

40. Elf J, Li G-W, Xie XS. Probing Transcription Factor Dynamics at the Single-Molecule Level in a Living Cell. Science (New York, N.Y.). 2007; 316(5828):1191-1194.

41. Friedman LJ, Gelles J. Mechanism of transcription initiation at an activator-dependent promoter defined by single-molecule observation. Cell. 2012; 148(4):679-689. [PubMed: 22341441]

42. Oehler S, Eismann ER, Kramer H, Müller-hill B. The three operators of the lac operon cooperate in repression. EMBO Journal. 1990; 9(4):973-979. [PubMed: 2182324]

43. Bremer H, Dennis PP. Modulation of Chemical Composition and Other Parameters of the Cell by Growth Rate. Escherichia coli and Salmonella: cellular and molecular biology. 1996; (122):15531569.

44. Klumpp S, Hwa T. Growth-rate-dependent partitioning of RNA polymerases in bacteria. Proceedings of the National Academy of Sciences of the United States of America. 2008; 105(51): 20245-20250. [PubMed: 19073937]

45. Vossen KM, Stickle DF, Fried MG. The mechanism of CAP-lac repressor binding cooperativity at the E. coli lactose promoter. Journal of molecular biology. 1996; 255(1):44-54. [PubMed: 8568874]

46. Johnson S, Lindén M, Phillips R. Sequence dependence of transcription factor-mediated DNA looping. Nucleic acids research. 2012; 40(16):7728-7738. [PubMed: 22718983]

47. Oehler S, Amouyal M, Wilcken-bergmann BV, Müller-hill B. Quality and position of the three lac operators of E. coli define efficiency of repression. EMBO Journal. 1994; 13(14):3348-3355. [PubMed: 8045263]

48. Poelwijk FJ, Kiviet DJ, Tans SJ. Evolutionary potential of a duplicated repressor-operator pair: simulating pathways using mutation data. PLoS computational biology. 2006; 2(5):e58. [PubMed: 16733549]

49. Santillán M. On the Use of the Hill Functions in Mathematical Models of Gene Regulatory Networks. Mathematical Modelling of Natural Phenomena. 2008; 3(2):85-97.

50. Martínez-Antonio A, Collado-Vides J. Identifying global regulators in transcriptional regulatory networks in bacteria. Current Opinion in Microbiology. 2003; 6(5):482-489. [PubMed: 14572541]

51. Dawid A, Kiviet DJ, Kogenaru M, de Vos M, Tans SJ. Multiple peaks and reciprocal sign epistasis in an empirically determined genotype-phenotype landscape. Chaos (Woodbury, N.Y.). 2010; 20(2):026105.

52. Kinney JB, Tkacik G, Callan CG. Precise physical models of protein-DNA interaction from highthroughput data. Proceedings of the National Academy of Sciences of the United States of America. 2007; 104(2):501-506. [PubMed: 17197415] 
(a) lac operon expression control knobs
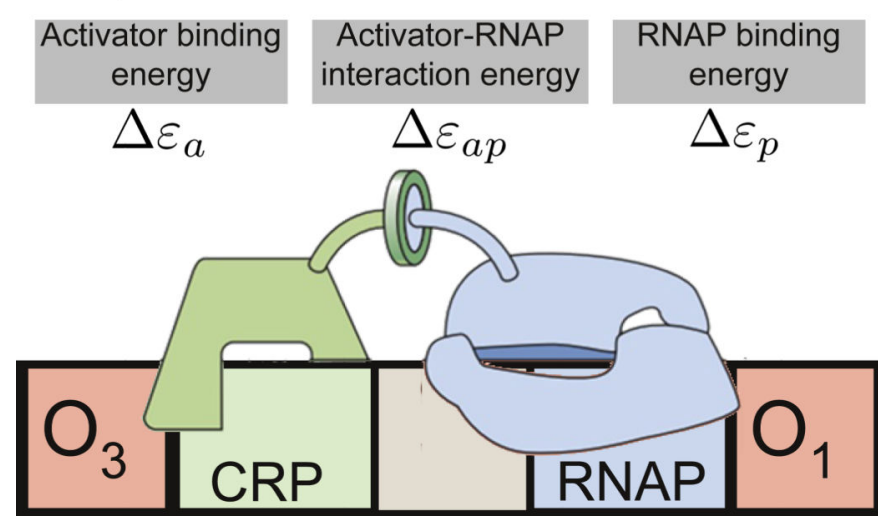

$\Delta \varepsilon_{p}$

Repressor binding energy

Activator-Repressor

Interaction

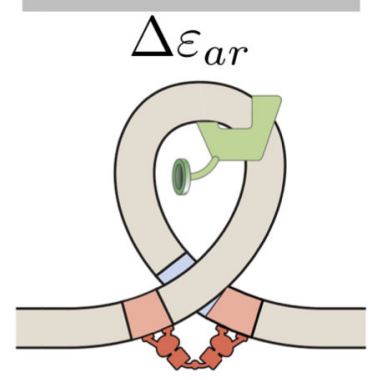

(b)
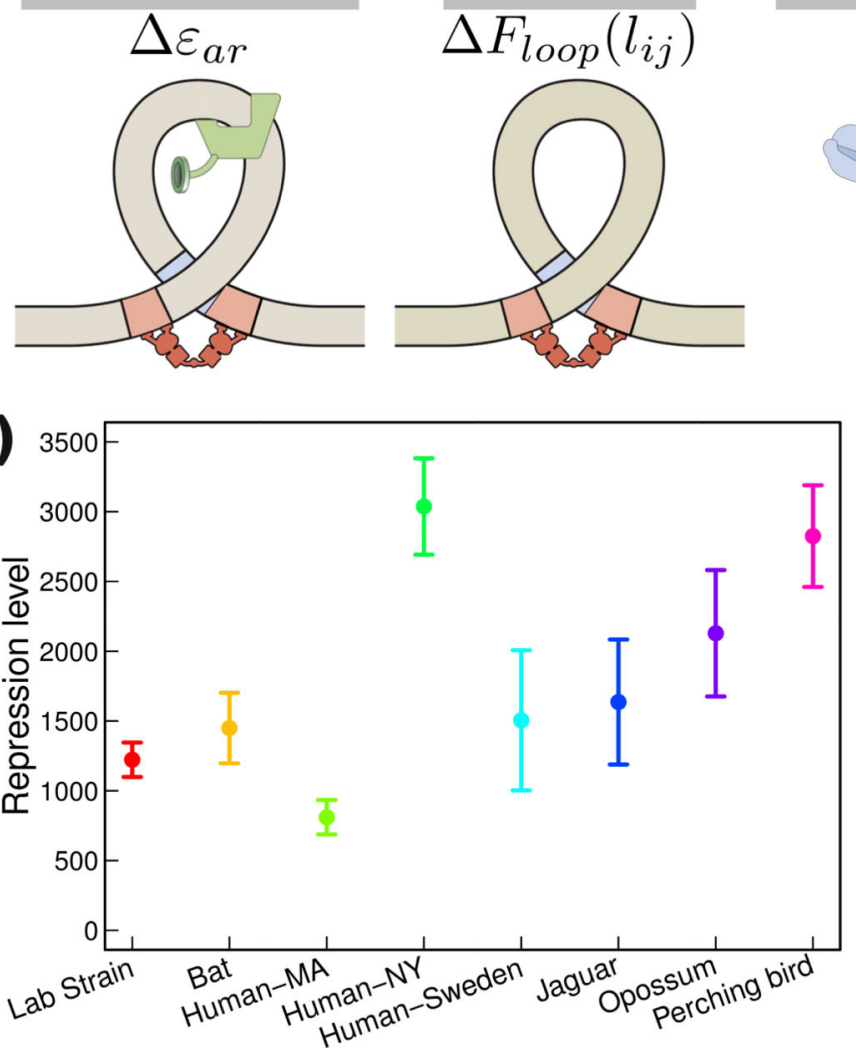

\section{RNAP copy}

number

$P$
Repressor copy number

$R$
$\Delta \varepsilon_{r}^{O_{i}}$
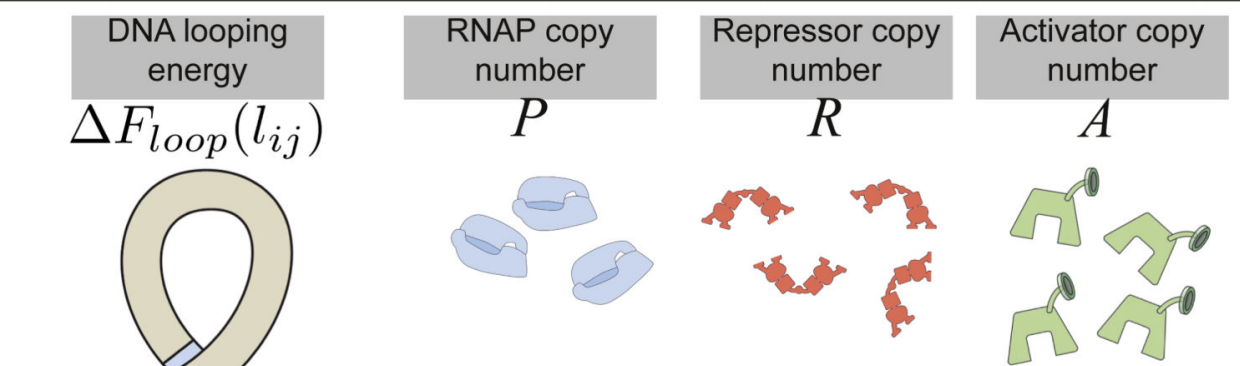

Repressor OFF, high expression of LacZ protein

(C) Fully induced cells [1 mM IPTG]

Repression

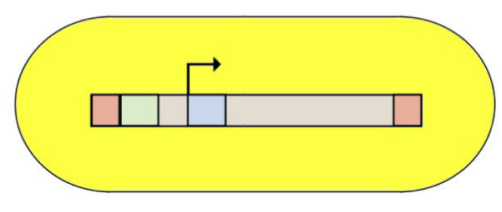

level $=$

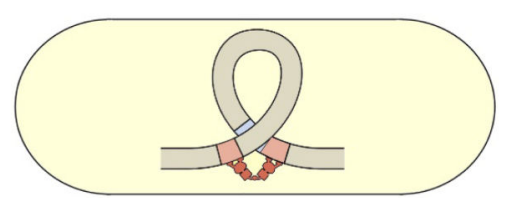

Uninduced cells [0 mM IPTG]

Repressor ON, low expression of LacZ protein

Figure 1.

(a) Regulatory knobs that control the expression of the lac operon and the symbols used to characterize these knobs in the thermodynamic model. The activator CRP increases expression, the Lac repressor binds to the three operators to decreases expression, and looping can lock the repressor onto $O_{1}$ leading to increased repression. The interaction energy between RNAP and CRP reflects the stabilization of the open complex formation due to the presence of the activator [28], and the interaction between the Lac repressor and CRP stabilizes the formation of the upstream loop [29]. (b) Variability in the repression level of E. coli natural isolates and the lab control strain MG1655. Strains are named after the host 
organism from which they were originally isolated [30]. Error bars represent the standard deviation from at least three independent measurements. (c) Schematic representation of the repression level, in which the role of the repressor in gene regulation is experimentally measured by comparing the ratio of LacZ proteins in cells grown in the presence of $1 \mathrm{mM}$ IPTG to cells grown in the absence of IPTG. LacZ protein concentrations were measured using a colorimetric assay. 


\begin{tabular}{|c|c|c|c|}
\hline State & Weight & State & Weight \\
\hline 国 & 1 & तर्राम & $\frac{A}{N_{N S}} e^{-\beta \Delta \varepsilon_{a}}$ \\
\hline पर्था म & $\frac{P}{N_{N S}} e^{-\beta \Delta \varepsilon_{p}}$ & 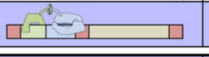 & $\frac{(A)(P)}{N_{N S}^{2}} e^{-\beta\left(\Delta \varepsilon_{a}+\Delta \varepsilon_{p}+\Delta \varepsilon_{a p}\right)}$ \\
\hline प्रि मे & $\frac{2 R(P)}{N_{N S}^{2}} e^{-\beta\left(\Delta \varepsilon_{r}^{O 2}+\Delta \varepsilon_{p}\right)}$ & लि को & $\frac{2 R(P)}{N_{N S}^{2}} e^{-\beta\left(\Delta \varepsilon_{r}^{O 3}+\Delta \varepsilon_{p}\right)}$ \\
\hline ति म & $\frac{4 R(R-1)(P)}{N_{N S}^{3}} e^{-\beta\left(\Delta \varepsilon_{r}^{O 2}+\Delta \varepsilon_{r}^{O 3}+\Delta \varepsilon_{p}\right)}$ & $\square+\frac{1}{4}$ & $\frac{2 R(A)(P)}{N_{N S}^{3}} e^{-\beta\left(\Delta \varepsilon_{a}+\Delta \varepsilon_{r}^{O 2}+\Delta \varepsilon_{p}+\Delta \varepsilon_{a p}\right)}$ \\
\hline अन्ति म & $\frac{2 R(A)(P)}{N_{N S}^{3}} e^{-\beta\left(\Delta \varepsilon_{a}+\Delta \varepsilon_{p}+\Delta \varepsilon_{r}^{O 3}\right)}$ & अधिक & $\frac{4 R(R-1)(A)(P)}{N_{N S}^{4}} e^{-\beta\left(\Delta \varepsilon_{a}+\Delta \varepsilon_{p}+\Delta \varepsilon_{r}^{O 2}+\Delta \varepsilon_{r}^{O 3}\right)}$ \\
\hline 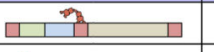 & $\frac{2 R}{N_{N S}} e^{-\beta \Delta \varepsilon_{r}^{O 1}}$ & पाम & $\frac{2 R}{N_{N S}} e^{-\beta \Delta \varepsilon_{r}^{O 2}}$ \\
\hline (11 & $\frac{2 R}{N_{N S}} e^{-\beta \Delta \varepsilon_{r}^{O 3}}$ & 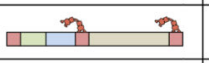 & $\frac{4 R(R-1)}{N_{N S}^{2}} e^{-\beta\left(\Delta \varepsilon_{r}^{O 1}+\Delta \varepsilon_{r}^{O 2}\right)}$ \\
\hline 舟, & $\frac{4 R(R-1)}{N_{N S}^{2}} e^{-\beta\left(\Delta \varepsilon_{r}^{O 1}+\Delta \varepsilon_{r}^{O 3}\right)}$ & का & $\frac{4 R(R-1)}{N_{N S}^{2}} e^{-\beta\left(\Delta \varepsilon_{r}^{O 2}+\Delta \varepsilon_{r}^{O 3}\right)}$ \\
\hline m n & $\frac{6 R(R-1)(R-2)}{N_{N S}^{3}} e^{-\beta\left(\Delta \varepsilon_{r}^{O 1}+\Delta \varepsilon_{r}^{O 2}+\Delta \varepsilon_{r}^{O 3}\right)}$ & 12 & $\frac{2 R(A)}{N_{N S}^{2}} e^{-\beta\left(\Delta \varepsilon_{a}+\Delta \varepsilon_{r}^{O 1}\right)}$ \\
\hline तf & $\frac{2 R(A)}{N_{N S}^{2}} e^{-\beta\left(\Delta \varepsilon_{a}+\Delta \varepsilon_{r}^{O 2}\right)}$ & af? & $\frac{4 R(R-1)(A)}{N_{N S}^{2}} e^{-\beta\left(\Delta \varepsilon_{a}+\Delta \varepsilon_{r}^{O 1}+\Delta \varepsilon_{r}^{O 2}\right)}$ \\
\hline$\left.M\right|^{211}$ & $\frac{2 R(A)}{N_{N S}^{2}} e^{-\beta\left(\Delta \varepsilon_{a}+\Delta \varepsilon_{r}^{O 3}\right)}$ & ân & $\frac{4 R(R-1)(A)}{N_{N S}^{2}} e^{-\beta\left(\Delta \varepsilon_{a}+\Delta \varepsilon_{r}^{O 1}+\Delta \varepsilon_{r}^{O 3}\right)}$ \\
\hline$M=11 \quad 7$ & $\frac{4 R(R-1)(A)}{N_{N S}^{2}} e^{-\beta\left(\Delta \varepsilon_{a}+\Delta \varepsilon_{r}^{O 2}+\Delta \varepsilon_{r}^{O 3}\right)}$ & and a & $\frac{6 R(R-1)(R-2)(A)}{N_{N S}^{4}} e^{-\beta\left(\Delta \varepsilon_{a}+\Delta \varepsilon_{r}^{O 1}+\Delta \varepsilon_{r}^{O 2}+\Delta \varepsilon_{r}^{O 3}\right)}$ \\
\hline (0) & $\frac{2 R}{N_{N S}} e^{-\beta\left(\Delta \varepsilon_{r}^{O 1}+\Delta \varepsilon_{r}^{O 2}+\Delta F_{l o o p}\left(l_{12}\right)\right)}$ & (0) & $\frac{2 R}{N_{N S}} e^{-\beta\left(\Delta \varepsilon_{r}^{O 1}+\Delta \varepsilon_{r}^{O 3}+\Delta F_{l o o p}\left(l_{13}\right)\right)}$ \\
\hline$=0$ & $\frac{2 R}{N_{N S}} e^{-\beta\left(\Delta \varepsilon_{r}^{O 2}+\Delta \varepsilon_{r}^{O 3}+\Delta F_{l o o p}\left(l_{23}\right)\right)}$ & (1) & $\frac{4 R(R-1)}{N_{N S}^{2}} e^{-\beta\left(\Delta \varepsilon_{r}^{O 1}+\Delta \varepsilon_{r}^{O 2}+\Delta \varepsilon_{r}^{O 3}+\Delta F_{l o o p}\left(l_{12}\right)\right)}$ \\
\hline$=0$ & $\frac{4 R(R-1)}{N_{N S}^{2}} e^{-\beta\left(\Delta \varepsilon_{r}^{O 1}+\Delta \varepsilon_{T}^{O 2}+\Delta \varepsilon_{r}^{O 3}+\Delta F_{l o o p}\left(l_{13}\right)\right)}$ & $=$ & $\frac{4 R(R-1)}{N_{N S}^{2}} e^{-\beta\left(\Delta \varepsilon_{r}^{O 1}+\Delta \varepsilon_{r}^{O 2}+\Delta \varepsilon_{r}^{O 3}+\Delta F_{l o o p}\left(l_{23}\right)\right)}$ \\
\hline (0) & $\frac{2 R(A)}{N_{N S}^{2}} e^{-\beta\left(\Delta \varepsilon_{r}^{O 1}+\Delta \varepsilon_{r}^{O 2}+\Delta \varepsilon_{a}+\Delta F_{l o o p}\left(l_{12}\right)\right)}$ & (1) & $\frac{4 R(R-1)(A)}{N_{N S}^{3}} e^{-\beta\left(\Delta \varepsilon_{r}^{O 1}+\Delta \varepsilon_{r}^{O 2}+\Delta \varepsilon_{r}^{O 3}+\Delta \varepsilon_{a}+\Delta F_{l o o p}\left(l_{12}\right)\right)}$ \\
\hline$=(90$ & $\frac{2 R(A)}{N_{N S}^{2}} e^{-\beta\left(\Delta \varepsilon_{r}^{O 1}+\Delta \varepsilon_{r}^{O 3}+\Delta \varepsilon_{a}+\Delta \varepsilon_{a r}+\Delta F_{l o o p}\left(l_{13}\right)\right)}$ & $=0$ & $\frac{4 R(R-1)(A)}{N_{N S}^{3}} e^{-\beta\left(\Delta \varepsilon_{r}^{O 1}+\Delta \varepsilon_{r}^{O 2}+\Delta \varepsilon_{r}^{O 3}+\Delta \varepsilon_{a}+\Delta \Delta \varepsilon_{a r}+\Delta F_{l o o p}\left(l_{13}\right)\right)}$ \\
\hline
\end{tabular}

Figure 2.

Thermodynamic mod1el of gene regulation. The table shows all states permitted within the model and their respective statistical weights as obtained using statistical mechanics. In these weights $P=$ number of RNAP per cell, $R=$ number of repressor molecules per cell, A $=$ number of activator molecules per cell, $\Delta \varepsilon_{r}^{O_{i}}=$ binding energy of Lac repressor to the $i^{\text {th }}$ operator, $\Delta \varepsilon_{p}=$ binding energy of RNA polymerase to the promoter, $\Delta \varepsilon_{a}=$ activator binding energy, $\Delta F_{\text {loop }}\left(l_{i j}\right)=$ looping free energy between operator $O_{i}$ and $O_{j}, N_{N S}=$ number of nonspecific binding sites on the genome, $\Delta \varepsilon_{a p}=$ interaction energy between the activator and the RNAP, $\Delta \varepsilon_{a r}=$ interaction energy between the activator and the repressor, and $\beta=$ inverse of the Boltzmann constant times the temperature (see Supplemental Material for further discussion). States with blue background are assumed to lead to transcription of the operon. 

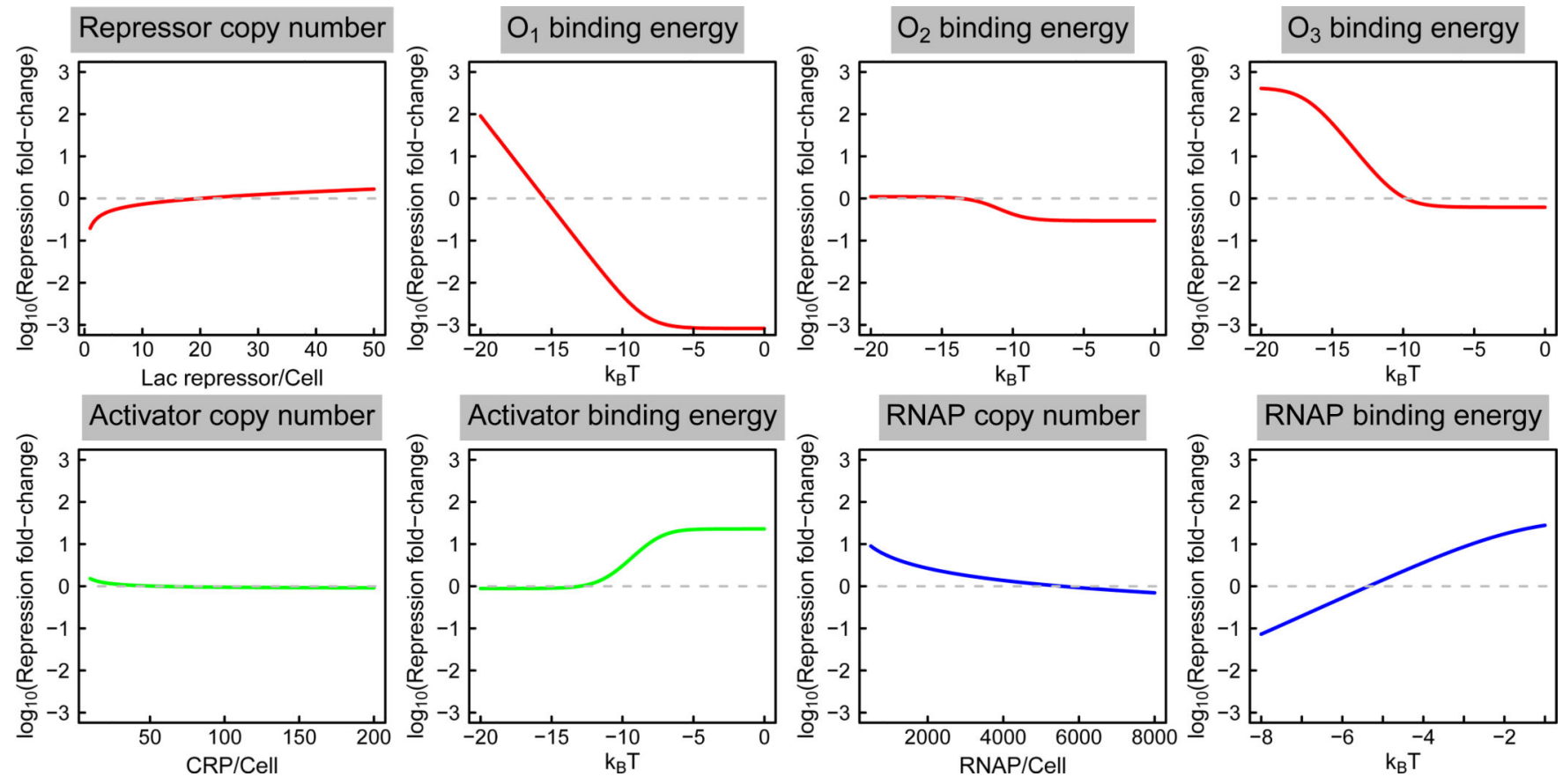

RNAP binding energy

Figure 3.

Sensitivity of phenotype to the parameters controlling the gene expression level. Each graph shows how a specific model parameter changes the level of gene expression. The $\log _{10}$ ratio of repression is calculated with respect to the predicted repression for the lab strain MG1655. The vertical axis spans between 1000 fold decrease to 1000 fold increase in repression with respect to this strain. The gray dotted line indicates the reference value for the lab strain MG1655. Values above this line indicate the operon is more tightly repressed and values below this line have a leakier expression profile (see Table S1 for further detail on the reference parameters). 
(a)

(b)
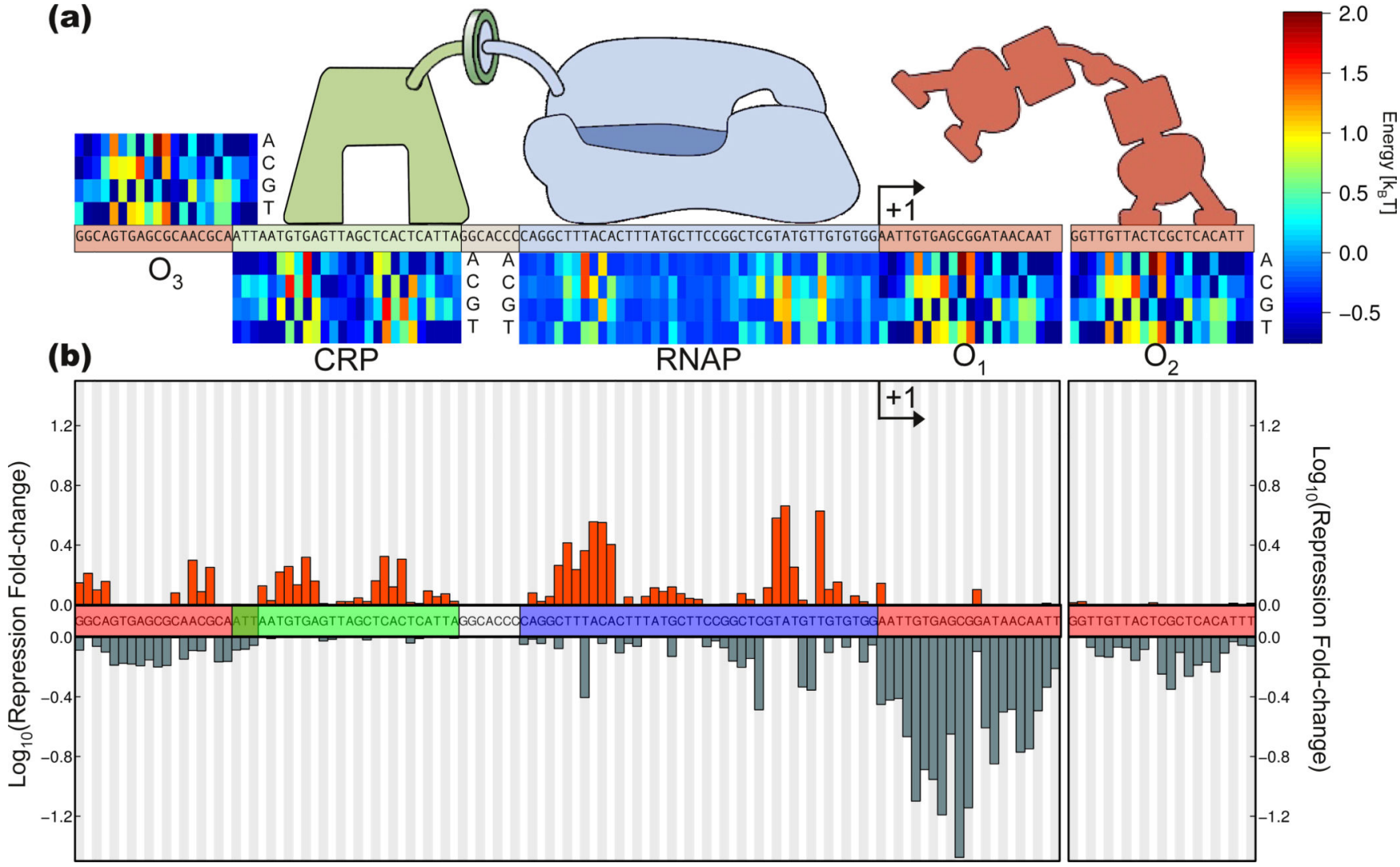

Figure 4.

Mapping from promoter sequence to regulatory level. (a) Energy matrices for the relevant transcription factors (Blue - RNAP, green - CRP, red - Lac repressor). These matrices allow us to map from sequence space to the corresponding binding energy. The contribution of each base pair to the total binding energy is color coded. The total binding energy for a given sequence is obtained by adding together the contribution of each individual base pair. (b) Using the energy matrices from (a) and the model whose states are depicted in Figure 2, the $\log _{10}$ repression change was calculated for all possible single point mutations of the promoter region. The height of the bars represents the biggest possible changes in the repression level (gray bars for biggest predicted decrease in repression, orange bar for biggest predicted increase in repression) given that the corresponding base pair is mutated with respect to the reference sequence (lac promoter region of the lab strain MG1655). The red arrows indicate the transcription start site. 


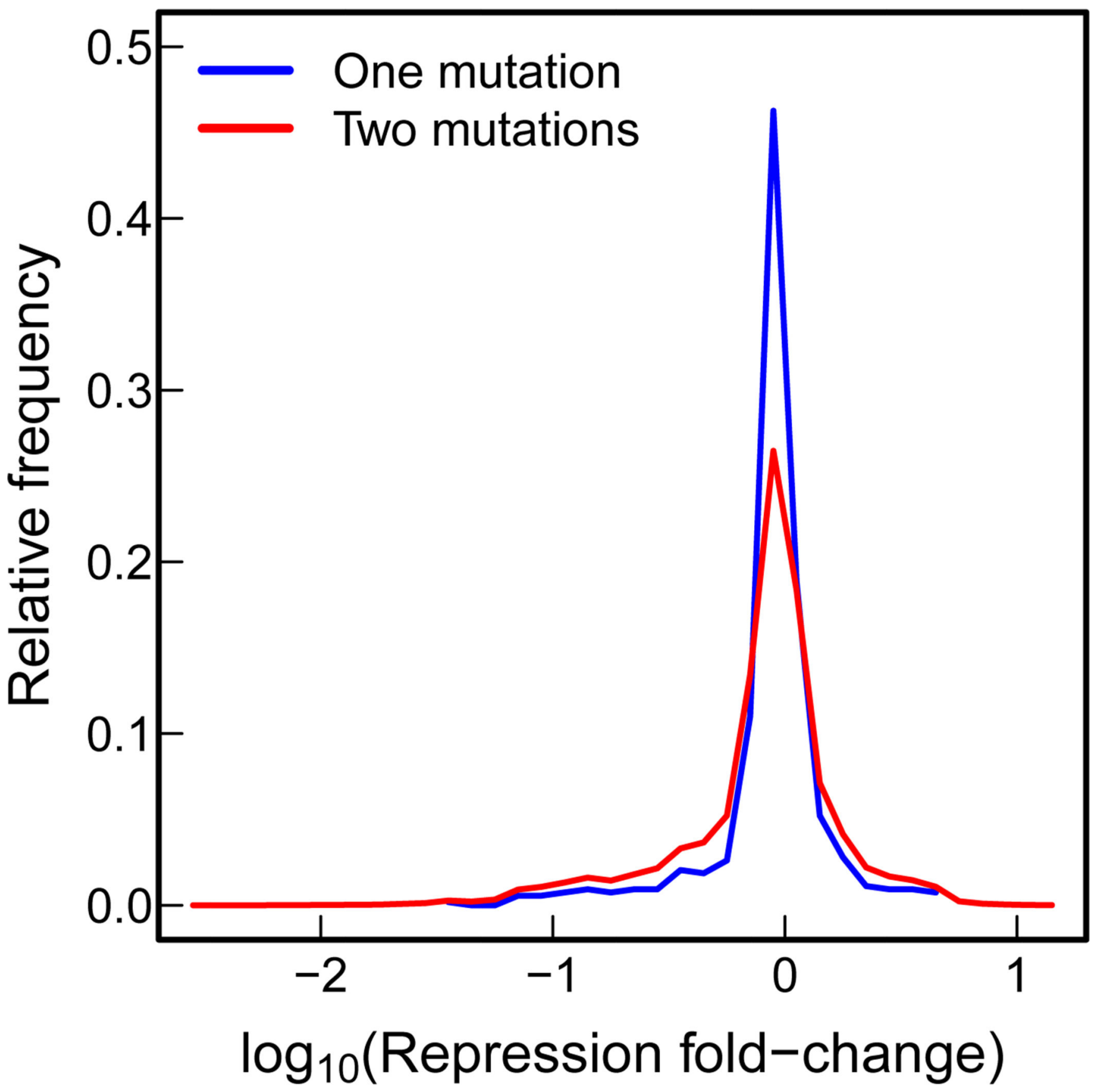

Figure 5.

Phenotype change distribution. Relative frequency of the predicted changes in repression level by mutating one (blue) or two (red) base pairs from the reference sequence (MG1655 promoter region). 
(a)
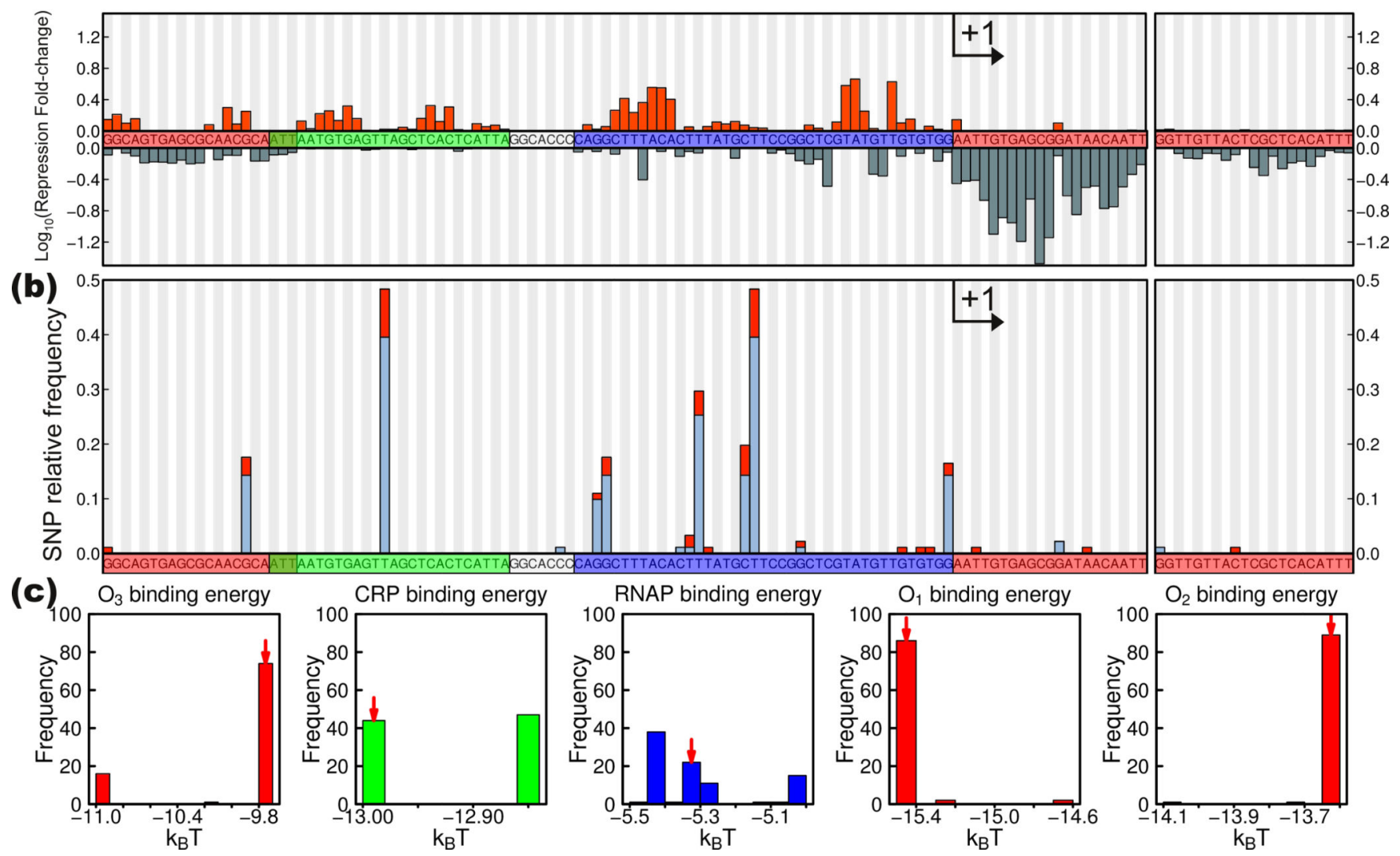

Figure 6.

Mutational landscape of the regulatory region of the lac operon. (a) The genotype to phenotype map is reproduced from Figure 4(b) in order to show how each base pair in the region influences gene regulation. (b) Comparing the sequence of the lac promoter from 91 E. coli strains identifies which base pairs were mutated in this region. The height of the bars represent the relative frequency of a mutation with respect to the consensus sequence. The red part of each bar represents the 22 natural isolates from different hosts [30] and the light blue part of these bars represents the 69 fully sequenced genomes (http:// www.ncbi.nlm.nih.gov/genomes/MICROBES/microbial_taxtree.html). Color coding of the binding sites and the transcription start site is as in Figure 4. (c) Using the energy matrices of Figure 4(a), we calculate the variability of protein binding energies for all sequences. The red arrow indicates reference binding energies for control strain MG1655. 


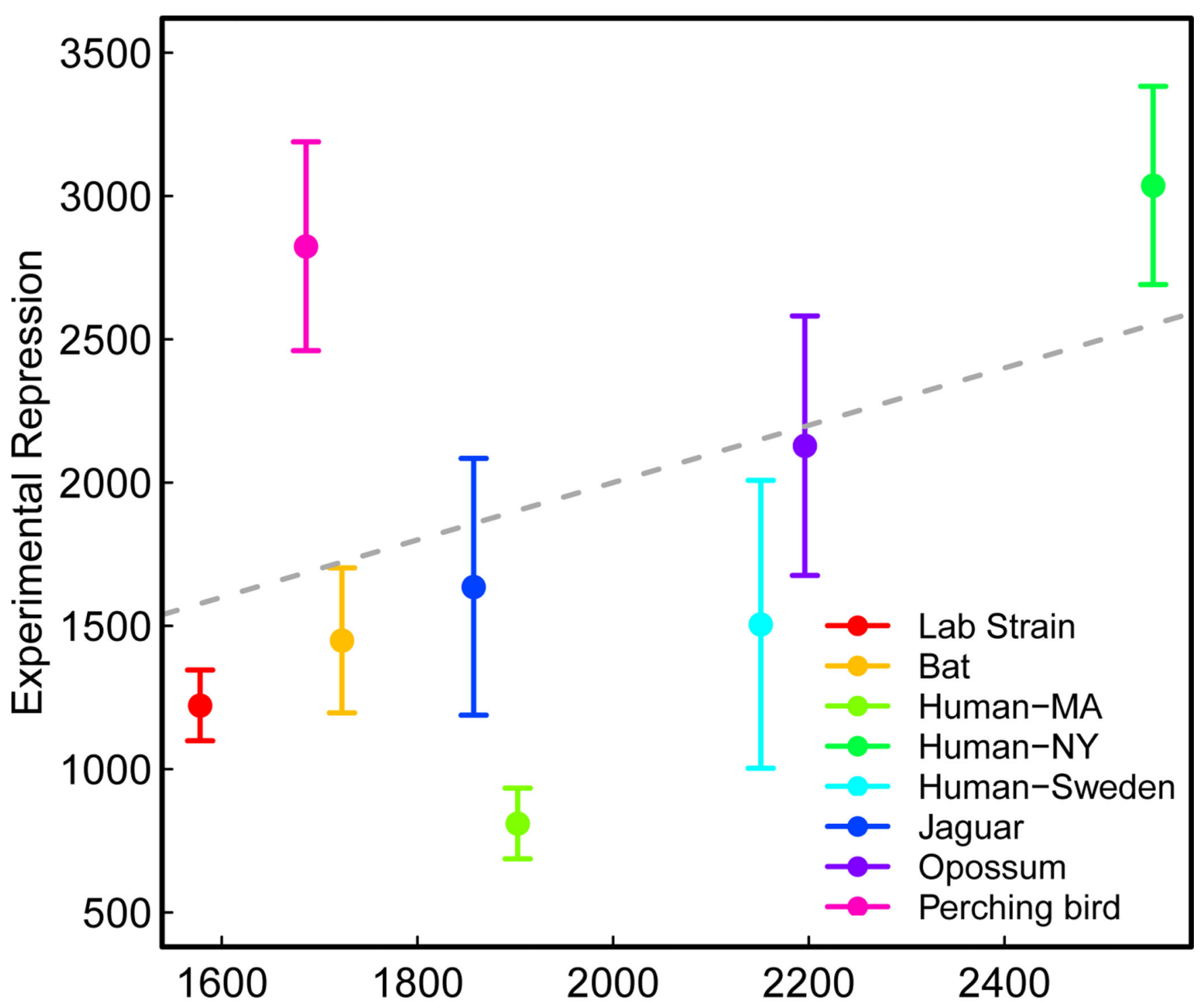

\section{Model Prediction}

Figure 7.

Comparison of model predictions with experimental measurements. Error bars represent the standard deviation of at least 3 independent measurements each with three replicates. The dotted line plots $x=y$. 
(a)

\section{S rRNA phylogenetic tree $\log _{10}$ Repression fold-change}

\section{Predicted}

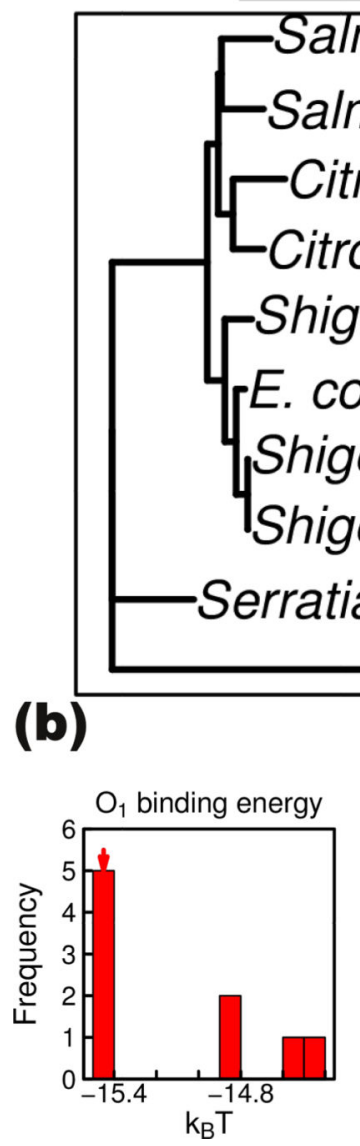

Salmonella enterica

Citrobacter rodentium

Citrobacter koseri

Shigella dysenteriae

E. coli MG1655

Shigella sonnei 53G

Shigella sonnei Ss046

Serratia marcescens

Vibrio Cholerae - 0.01
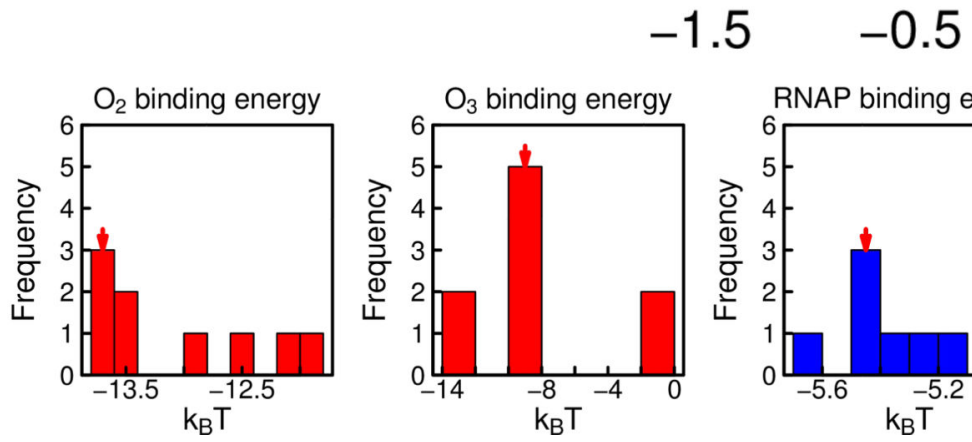

1.5

Figure 8.

Predicted variability among different microbial species based on genome sequences and our model for regulation derived for E. coli. (a) On the left a 16S rRNA phylogenetic tree of diverse species with a similar lac promoter architecture done with the Neighbor-Joining algorithm. Vibrio cholerae was used as an outgroup species. The scale bar represents the relative number of substitutions per sequence. On the right the predicted $\log _{10}$ fold-change in repression with respect to $E$. coli MG1655 assuming the same growth rate and transcription factor copy numbers. The outgroup species fold-change was not calculated. (b) Parameter distribution calculated using the promoter region sequence and the energy matrices. The red arrow indicates the MG1655 reference value. Strains lacking a binding site were binned as zero. 


\section{Table 1}

Lac repressor copy number as measured with the immunodot blots and doubling time of the eight strains with measured repression level shown in Figure 1(b). The errors represent the standard error of 3 independent experiments.

\begin{tabular}{lll}
\hline Strain & Repressor/cell & Doubling time [min] \\
\hline Lab strain & $21 \pm 4$ & $29.1 \pm 0.2$ \\
Bat & $12 \pm 1$ & $27.5 \pm 0.2$ \\
Human-MA & $20 \pm 4$ & $35.6 \pm 0.6$ \\
Human-NY & $23 \pm 4$ & $41.5 \pm 0.4$ \\
Human-Sweden & $28 \pm 1$ & $34.2 \pm 0.3$ \\
Jaguar & $21 \pm 3$ & $32.0 \pm 0.2$ \\
Opossum & $26 \pm 2$ & $33.5 \pm 0.2$ \\
Perching bird & $24 \pm 4$ & $30.2 \pm 0.3$ \\
\hline
\end{tabular}

